\title{
Ação de Polistes (Aphanilopterus) simillimus Zikán (Hymenoptera, Vespidae) no controle de Spodoptera frugiperda (Smith) (Lepidoptera, Noctuidae)
}

\author{
Fábio Prezoto ${ }^{1}$ \\ Vera L.L. Machado ${ }^{2}$
}

\begin{abstract}
Action of Polistes (Aphanilopterus) simillimus Zikán (Hymenoptera, Vespidae) in the control of Spodoptera frugiperda (Smith) (Lepidoptera, Noctuidae). The corn is a plantation attacked by a lot of pests, mainly by caterpillar Spodoptera frugiperda (Smith), producing serious damages in the plants, occasioning unproductiveness. Thus, the goal of this research was to verify the predation of Polistes simillimus (Zikán, 1951) wasps on this pest of a corn plantation using the wasps colonies as a efficient form of this pest management. The present research was developed in a area in the Piracicaba county, São Paulo ( $\left.22^{\circ} 43^{\prime} \mathrm{S}, 47^{\circ} 38^{\prime} \mathrm{W}\right)$, during the period of november/1995-march/1996, introducing $20 \mathrm{P}$. simillimus colonies in artificial wood shelters around of 5 plots of corn, to accompany the foraging activity of the wasps and the development of the wasps colonies. Spodoptera frugiperda $(23,07 \%)$ was the main prey captured by the wasps and the average rate of capture was $1,54 \mathrm{preys} / \mathrm{h}$. These data gave an estimation of reduction in the occurrence of $S$. frugiperda $(77,16 \%)$, due mainly to the wasps action.
\end{abstract}

KEY WORDS. Biology control, corn plantation, pests and predators

A predação de Polistes principalmente sobre lagartas de lepidópteros é mencionada desde 1905 por PECKHAMS (apud GILLASPY 1979). Subseqüentemente surgiram muitas informações sobre a manipulação e predação de Polistes sobre pragas de ocorrência natural.

Na Carolina do Norte, RABB \& LAWSON (1957) verificaram o potencial das vespas como agentes de controle biológico de pragas, pela introdução de colônias de $P$. exclamans Viereck, 1906 e $P$. fuscatus (Fabricius, 1793) na cultura do fumo, as quais reduziram em $68 \%$ o dano causado pela lagarta Protoparce sexta (Cramer, 1779) (Lepidoptera, Sphingidae). Também na Carolina do Norte, RABB (1960) comprovou que mais de $95 \%$ das presas de $P$. fuscatus, $P$. exclamans e $P$. annularis (Linnaeus, 1763) eram lagartas de lepidópteros.

A eficiência das vespas como inimigos naturais de pragas de culturas também foi estudada por MORIMOTO (1960a,b, 1961), que verificou que uma única colônia de Polistes utiliza 2000 lagartas de Pieris rapae (Linnaeus, 1764) (Lepidoptera, Pieridae) durante seu desenvolvimento, tornando viável o controle de pragas pela introdução de colônias de Polistinae.

A fim de facilitar o manejo de colônias de Polistes, muitos pesquisadores têm testado vários tipos de abrigos ao redor de culturas (KIRKTON 1970; GILLASPY

1) Departamento de Zoologia, Instituto de Ciências Biológicas, Universidade Federal de Juiz de Fora. Campus Universitário, 36033-330 Juiz de Fora, Minas Gerais, Brasil.

2) Centro de Estudos sobre Insetos Sociais, Instituto de Biociências, Universidade Estadual Paulista. Caixa postal 199, 13506-900 Rio Claro, São Paulo, Brasil. 
1970, 1971a,b, 1972, 1973a,b). Mais recentemente, TURILLAZZI (1980) testou com sucesso a adoção de ninhos artificiais feitos com cápsulas de gelatina, cera, plástico e tubos de vidro para algumas espécies de Polistes. Desta forma, a introdução de colônias de vespas numa cultura pode trazer benefícios tanto para a lavoura, pela redução da praga, como para a colônia de vespas, pela oferta de alimento e abrigo.

Dentre os inúmeros fatores que afetam a produtividade do milho, destacamse as pragas e, entre estas, a lagarta do cartucho Spodoptera frugiperda (J.E. Smith, 1797) (Lepidoptera, Noctuidae) é que tem a maior importância, não somente pelos danos causados, mas também pela dificuldade do seu controle (BolçA et al. 1992).

CARVAlHO (1970 apud NAKANO et al. 1981) constatou que os danos causados por esta praga são variáveis, dependendo do estágio vegetativo da planta e que plantas com o cartucho bastante danificado sofrem perda da produção na ordem de 15 a $35 \%$ aos 35 dias; 29 a $60 \%$ aos 49 dias e $34,1 \%$ aos 60 dias após a semeadura. Os dados de CARVALHO (1970 apud NAKANO et al. 1981) vão de encontro aos obtidos por BoIÇA et al. (1992) em que os autores afirmam que as plantas entre 35 a 45 dias são mais prejudicadas pelos ataques de $S$. frugiperda, comprometendo a produção.

Desta forma, o presente trabalho teve como finalidade avaliar a ação predatória de $P$. simillimus sobre lagartas de $S$. frugiperda em milho no campo, verificando-se os efeitos do controle desta praga para a cultura e vespas.

\section{MATERIAL E MÉTODOS}

O presente experimento foi realizado no sítio Prezoto, município de Piracicaba, São Paulo ( $\left.22^{\circ} 43^{\prime} \mathrm{S}, 47^{\circ} 38^{\prime} \mathrm{W}\right)$, durante o período de novembro de 1995 a março de 1996, em uma área de $700 \mathrm{~m}^{2}$. Nesta área foram distribuídas 20 colônias de vespas, segundo a distribuição de Poisson, em 5 quadras (Q1, Q2, Q3, Q4 e Q5) de milho de $10 \times 10$ metros $\left(500 \mathrm{~m}^{2}\right)$. Em outra área distante 750 metros e separada por talhões de cana-de-açúcar foram estabelecidas mais duas quadras controles (Q6 e Q7, $200 \mathrm{~m}^{2}$ ), como referencial para o cálculo de incidência de pragas.

As cinco quadras estavam separadas entre si por uma distância de 8 metros, onde foram instalados os abrigos artificiais de madeira em forma de telhado, formado por duas tábuas de $15 \times 20 \times 1 \mathrm{~cm}$, presas a um sarrafo de 1,8 metros. Cada abrigo recebeu um pedaço de calha recobrindo a madeira para protegê-lo da chuva e do sol. Na base de cada sarrafo, cerca de 50 centímetros receberam proteção com óleo queimado e graxa, para se evitar ataque de formigas. Os abrigos foram distribuídos um em cada lado das cinco quadras infestadas, a uma distância de dois metros do limite da cultura, totalizando 20 ninhos de $P$. simillimus.

Seguindo-se a orientação da Casa da Agricultura, no plantio do milho utilizou-se o híbrido AL-34, que é rústico e resistente às condições climáticas da região. Foi adotado o espaçamento de 0,9 metros entre linhas e 0,25 metros entre plantas, perfazendo um total de 11 linhas por quadra.

Após o plantio, procedeu-se à translocação de colônias de $P$. simillimus para o local do experimento. As colônias foram coletadas no Horto Florestal "Navarro de Andrade", no município de Rio Claro e em diversas localidades no município de Piracicaba, ambos no estado de São Paulo, Brasil. 
Seguindo a metodologia de translocação de RICHARDS \& RICHARDS (1951), as colônias foram capturadas logo no início da noite com o auxílio de um saco plástico grande, ensacando-as cuidadosamente sem destruir os pedúnculos das mesmas. Após a retirada das colônias, estas foram acondicionadas em uma caixa de isopor e transportadas até o local do experimento. Em seguida, as colônias foram imediatamente coladas nos abrigos através de seu pedúnculo, seguindo-se a orientação anterior e, usando-se cola à base de éster de cianoacrilato (Super-bonder ${ }^{\mathbb{B}}$ ). O saco plástico contendo os indivíduos foi suspenso na parte inferior do abrigo durante a noite da translocação e retirado no dia seguinte depois que todas as vespas tinham deixado o mesmo.

As colônias translocadas $(n=20)$ foram denominadas alfabeticamente de A, $\mathrm{B}, \mathrm{C} \ldots$ até $\mathrm{U}$.

Após as translocações, iniciou-se o trabalho de quantificação da ação predatória das vespas sobre as pragas da cultura do milho. Essa quantificação foi realizada através da coleta do material trazido pelas vespas para suas colônias, durante uma hora por semana em cinco colônias, localizadas uma em cada quadra do experimento. Esta coleta consistiu em capturar com auxílio de uma rede entomológica, as vespas que retornavam ao ninho transportando material, facilmente reconhecidas devido ao vôo lento e/ou pelo volume da carga trazida. Imediatamente após a coleta do material transportado pelas vespas, este foi pesado e fixado em álcool a $70 \% \mathrm{e}$ identificado até o táxon possível.

Ainda com respeito a atividade forrageadora das vespas, foram quantificados os retornos com polpa de madeira, néctar e água. Os retornos sem carga foram desconsiderados. A identificação dos retornos com polpa de madeira se deu pela visibilidade do volume do mesmo trazido pela vespa, enquanto que as coletas de líquido foram identificadas pelo comportamento da vespa ao chegar ao ninho e assim, considerou-se retorno com néctar quando houve trofaláxis adulto-adulto ou adulto-larva e, retorno com água, quando o líquido foi depositado diretamente na parede das células, segundo a metodologia indicada por PREZOTO et al. (1994).

Além das coletas de presas, também foram tomadas informações sobre todas as colônias de vespas nos abrigos, através do mapeamento semanal de cada ninho.

Já em relação à cultura de milho, semanalmente foram dissecadas totalmente cinco plantas por quadra, a fim de se verificar a ocorrência dos insetos associados a ela e de se calcular a porcentagem da ação das vespas no controle das pragas. Com base no número médio de lagartas de $S$. frugiperda encontradas nas 5 plantas amostradas, estimou-se através de uma regra de três a quantidade de lagartas existentes no total de plantas (880) das quadras e dos controles. A variação das quadras experimento em relação às quadras controle foi transformada em porcentagem e o seu valor interpretado como sendo a estimativa da ação das vespas sobre as lagartas.

\section{RESULTADOS E DISCUSSÃO}

Foram realizadas 12 coletas semanais para se avaliar a incidência natural de insetos nas quadras experimento (Q1, Q2, Q3, Q4 e Q5) e controles (Q6 e Q7), referentes ao período de 22-XII-1995 a 8-III-1996, a fim de estabelecer um parâmetro com o material predado pelas vespas. 
A coleta de insetos nas quadras (experimento e controles) revelaram que a principal praga presente na cultura do milho foi a lagarta S. frugiperda (Tabs I, II). ZUCCHI et al. (1993) afirmam que essa lagarta é a principal praga do milho e que uma infestação média desta espécie reduz a produção em $20 \%$. Neste trabalho foram encontrados espécimens em quase todas as partes das plantas (exceto na raiz) apresentando-se nos diversos ínstares, além de serem freqüentemente coletados nas quadras experimento e controles (exceto na coleta 11 das quadras experimento). Outros insetos presentes estão relacionados nas tabelas I e II.

Tabela I. Registro dos insetos encontrados nas quadras experimento (Q1, Q2, Q3, Q4 e Q5) da cultura do milho durante as coletas semanais. (X) Presente, ( - ) ausente.

\begin{tabular}{|c|c|c|c|c|c|c|c|c|c|c|c|c|}
\hline \multirow{2}{*}{ Insetos Coletados } & \multicolumn{12}{|c|}{ Número de coleta semanal } \\
\hline & 1 & 2 & 3 & 4 & 5 & 6 & 7 & 8 & 9 & 10 & 11 & 12 \\
\hline \multicolumn{13}{|l|}{ Dermaptera } \\
\hline \multicolumn{13}{|l|}{ Forficulidae } \\
\hline Doru luteipes Scudder, 1876 & $x$ & $x$ & $x$ & $x$ & $x$ & $x$ & $x$ & $x$ & $x$ & $x$ & $x$ & $x$ \\
\hline \multicolumn{13}{|l|}{ Lepidoptera } \\
\hline \multicolumn{13}{|l|}{ Noctuidae } \\
\hline Spodoptera frugiperda (Smith, 1797) & $x$ & $x$ & $x$ & $x$ & $x$ & $x$ & $\mathrm{x}$ & $x$ & $x$ & $x$ & - & $x$ \\
\hline Spodoptera latifascia Walker, 1856 & - & - & - & - & - & - & - & - & - & - & $x$ & - \\
\hline Helicoverpa zea (Bod., 1850) & - & - & - & - & - & - & - & - & - & - & $\mathrm{x}$ & - \\
\hline \multicolumn{13}{|l|}{ Pyralidae } \\
\hline Elasmopalpus lignosellus (Zeller, 1848) & - & - & - & - & - & - & - & - & - & - & $x$ & - \\
\hline \multicolumn{13}{|l|}{ Coleoptera } \\
\hline \multicolumn{13}{|l|}{ Chrysomelidae } \\
\hline Diabrotica speciosa (Germar, 1824) & - & - & - & $x$ & $\mathrm{x}$ & $x$ & $x$ & - & $x$ & $x$ & $x$ & - \\
\hline Diabrotica sp. & - & - & $x$ & - & - & - & - & - & - & - & - & - \\
\hline Sternocolaspis quatuordecimcostata (Lefèvre, 1877) & $x$ & $\mathrm{x}$ & - & - & $x$ & $x$ & - & $\mathrm{x}$ & - & - & - & - \\
\hline \multicolumn{13}{|l|}{ Coccinellidae } \\
\hline Cycloneda sanguinea (Linnaeus, 1763) & - & $\mathrm{x}$ & $x$ & - & - & $x$ & - & - & - & - & - & - \\
\hline \multicolumn{13}{|l|}{ Elateridae } \\
\hline Conoderus sp. & - & $\mathrm{x}$ & - & $x$ & - & - & - & - & - & - & - & - \\
\hline \multicolumn{13}{|l|}{ Curculionidae } \\
\hline Pantomorus sp. & $x$ & $\mathrm{x}$ & $x$ & $x$ & - & - & $x$ & - & - & - & - & - \\
\hline Sitophilus zeamais Mots., 1865 & - & - & - & - & - & - & - & - & - & - & - & $x$ \\
\hline Nitidulidae & $x$ & - & $x$ & - & - & $x$ & - & - & - & - & - & - \\
\hline Carabidae & - & - & - & $\mathrm{x}$ & - & - & - & - & - & - & - & - \\
\hline \multicolumn{13}{|l|}{ Hemiptera } \\
\hline \multicolumn{13}{|l|}{ Coreidae } \\
\hline Leptoglossus zonatus (Dallas, 1852) & $\mathrm{x}$ & - & - & - & - & $x$ & $\mathrm{x}$ & $x$ & $x$ & $x$ & $\mathrm{x}$ & - \\
\hline \multicolumn{13}{|l|}{ Pyrrhocoridae } \\
\hline Euryophthalmus humilis (Drury, 1782) & - & - & $x$ & - & - & - & - & - & - & - & - & - \\
\hline \multicolumn{13}{|l|}{ Lygaeidae } \\
\hline Blissus leucopterus (Say, 1832) & - & - & $x$ & - & - & - & - & - & - & - & - & - \\
\hline \multicolumn{13}{|l|}{ Diptera } \\
\hline \multicolumn{13}{|l|}{ Otitidae } \\
\hline Euxesta sp. & $x$ & - & - & - & - & - & $\mathrm{x}$ & $x$ & - & $\mathrm{x}$ & $\mathrm{x}$ & - \\
\hline \multicolumn{13}{|l|}{ Stratiomyidae } \\
\hline Hermetia illucens Linnaeus, 1758 & - & - & - & - & $x$ & - & - & - & - & - & - & - \\
\hline \multicolumn{13}{|l|}{ Homoptera } \\
\hline Aphididae & & & & & & & & & & & & \\
\hline Rhopalosiphum maidis (Fitch., 1856) & - & $x$ & $x$ & $x$ & - & $x$ & - & $x$ & - & $x$ & $x$ & $x$ \\
\hline Thysanoptera & & & & & & & & & & & & \\
\hline Thripidae & - & $\mathrm{x}$ & - & - & - & - & - & - & - & - & - & - \\
\hline Isoptera & & & & & & & & & & & & \\
\hline Termitidae & & & & & & & & & & & & \\
\hline Embiratermes heterotypus (Silvestri, 1901) & - & - & - & - & - & - & $\mathrm{x}$ & - & $x$ & - & - & - \\
\hline
\end{tabular}

Revta bras. Zool. 16 (3): 841 - 850, 1999 
Tabela II. Registro dos insetos encontrados nas quadras controles (Q6 e Q7) da cultura do milho durante as coletas semanais $(X)$ Presente, $(-)$ ausente.

\begin{tabular}{|c|c|c|c|c|c|c|c|c|c|c|c|c|}
\hline \multirow{2}{*}{ Insetos Coletados } & \multicolumn{12}{|c|}{ Número de coleta semanal } \\
\hline & 1 & 2 & 3 & 4 & 5 & 6 & 7 & 8 & 9 & 10 & 11 & 12 \\
\hline \multicolumn{13}{|l|}{ Dermaptera } \\
\hline \multicolumn{12}{|l|}{ Doru luteipes Scudder, 1876} & $x$ \\
\hline \multicolumn{13}{|l|}{ Lepidoptera } \\
\hline \multicolumn{13}{|l|}{ Noctuidae } \\
\hline Spodoptera frugiperda (Smith, 1797) & $x$ & $x$ & $x$ & $x$ & $\mathrm{x}$ & $\mathrm{x}$ & $x$ & $\mathrm{x}$ & $\mathrm{x}$ & $\mathrm{x}$ & $\mathrm{x}$ & $x$ \\
\hline Helicoverpa zea (Bod., 1850) & - & - & - & - & - & - & - & - & - & - & - & $x$ \\
\hline \multicolumn{13}{|l|}{ Coleoptera } \\
\hline \multicolumn{13}{|l|}{ Chrysomelidae } \\
\hline Diabrotica speciosa (Germar, 1824) & - & - & $\mathrm{x}$ & - & - & - & $x$ & $x$ & $x$ & $\mathrm{x}$ & $x$ & - \\
\hline Diabrotica sp. & $x$ & - & $\mathrm{x}$ & $\mathrm{x}$ & - & $\mathrm{x}$ & - & - & - & - & - & - \\
\hline Sternocolaspis quatuordecimcostata (Lefèvre, 1877) & - & $\mathrm{x}$ & $x$ & - & - & - & - & - & - & - & - & - \\
\hline \multicolumn{13}{|l|}{ Elateridae } \\
\hline Conoderus sp. & $x$ & - & - & $x$ & - & - & - & - & - & - & - & - \\
\hline \multicolumn{13}{|l|}{ Curculionidae } \\
\hline Pantomorus sp. & $x$ & $x$ & $x$ & - & $x$ & - & $x$ & - & - & - & - & - \\
\hline Sitophilus zeamais Mots., 1865 & - & - & - & - & - & - & - & - & - & - & - & $x$ \\
\hline \multicolumn{13}{|l|}{ Nitidulidae } \\
\hline \multicolumn{13}{|l|}{ Lagriidae } \\
\hline Lagria villosa Fabricius, 1783 & - & - & - & - & - & $x$ & - & - & - & - & $x$ & - \\
\hline Carabidae & $x$ & - & $x$ & - & - & $x$ & - & - & - & - & - & - \\
\hline \multicolumn{13}{|l|}{ Hemiptera } \\
\hline \multicolumn{13}{|l|}{ Coreidae } \\
\hline Leptoglossus zonatus (Dallas, 1852) & - & - & - & - & - & - & $x$ & $x$ & $x$ & $x$ & $x$ & - \\
\hline Ploiariidae & - & - & $x$ & $x$ & - & - & - & - & - & - & - & - \\
\hline \multicolumn{13}{|l|}{ Diptera } \\
\hline \multicolumn{13}{|l|}{ Otitidae } \\
\hline Euxesta sp. & - & - & - & - & - & - & $x$ & - & $x$ & $\mathrm{x}$ & $x$ & - \\
\hline \multicolumn{13}{|l|}{ Stratiomyidae } \\
\hline Hermetia illucens Linnaeus, 1758 & - & - & $x$ & $x$ & - & - & - & - & - & - & - & - \\
\hline \multicolumn{13}{|l|}{ Homoptera } \\
\hline \multicolumn{13}{|l|}{ Aphididae } \\
\hline Rhopalosiphum maidis (Fitch., 1856) & - & - & $x$ & $x$ & $x$ & $x$ & $x$ & $x$ & $x$ & $x$ & $x$ & - \\
\hline
\end{tabular}

Através da identificação possível, as principais pragas capturadas por $P$. simillimus, na cultura do milho foram: S. frugiperda (23,07\%); Heliothis virescens (Fabricius, 1781) (15,38\%) e Anticarsia gemmatalis Huebner, 1818 (14,28\%), dentre outras (Tab. III). Trabalhando com a mesma espécie de vespa em região canavieira, PREZOTO et al. (1994) também encontraram as mesmas presas mas, em porcentagens diferentes: $S$. frugiperda $(2,1 \%) ; H$. virescens $(18,7 \%) \mathrm{e}$ A. gemmatalis $(4,2 \%)$. RABB (1960) encontrou uma variação na composição das presas capturadas por $P$. exclamans, durante o final de julho e início de agosto, quando as vespas capturaram mais lagartas de $S$. frugiperda do que de $H$. virescens, já em meados de agosto até início de setembro a quantidade foi inversa. As coletas realizadas na área de estudo indicaram que Helicoverpa zea (Bod., 1850) foi mais abundante que Heliothis virescens, contudo as lagartas de $H$. zea estavam presentes na espiga do milho e por isso menos expostas aos predadores.

Na figura 1 pode-se observar que o número médio das lagartas de $S$. frugiperda e Spodoptera latifascia Walk, 1856 nas quadras experimento e controles sempre foi menor nas primeiras, evidenciando a ação predatória das vespas na redução destes lepidópteros. 
Tabela III. Quantidade de presas capturadas por cinco colônias de Polistes simillimus durante cinco horas semanais (60 horas) na área eXperimental de uma cultura de milho.

\begin{tabular}{|c|c|c|c|c|c|c|c|c|c|c|c|c|c|}
\hline \multirow{2}{*}{ Presas capturadas } & \multicolumn{12}{|c|}{ Observaçס̄es semanais } & \multirow[t]{2}{*}{ Total( $\%)$} \\
\hline & 1 & 2 & 3 & 4 & 5 & 6 & 7 & 8 & 9 & 10 & 11 & 12 & \\
\hline \multicolumn{14}{|l|}{ Lepidoptera } \\
\hline Spodoptera frugiperda (Smith, 1797) & 4 & - & 1 & 1 & 2 & 1 & 3 & 1 & 1 & 1 & 2 & 3 & 23,07 \\
\hline Spodoptera latifascia Walker, 1856 & - & - & - & - & - & - & - & 1 & - & - & - & - & 1,09 \\
\hline Heliothis virescens (Fabricius, 1781) & 1 & 3 & - & 2 & 3 & 1 & 2 & - & 1 & - & 1 & - & 15,38 \\
\hline Helicoverpa zea (Bod., 1850) & - & 1 & 2 & - & - & - & - & 1 & 1 & 1 & 2 & 2 & 10,98 \\
\hline Mocis latipes (Guenée, 1852) & - & - & 1 & - & - & - & - & - & - & - & - & - & 1,09 \\
\hline Trichoplusia ni (Huebner, 1802) & - & - & 2 & 3 & - & - & - & - & - & 2 & 1 & - & 8,79 \\
\hline Anticarsia gemmatalis Huebner, 1818 & - & - & - & - & - & 2 & - & 3 & 2 & 2 & 3 & 1 & 14,28 \\
\hline Nymphalidae & - & - & - & - & - & 2 & - & - & - & - & - & - & 2,19 \\
\hline Pieridae & - & - & - & - & - & - & 1 & - & - & - & - & - & 1,09 \\
\hline Năo identificados & - & - & 1 & - & - & - & - & 3 & 2 & 2 & 3 & - & 12,08 \\
\hline Ninfa de Hemiptera & - & - & - & - & - & - & - & - & - & 1 & - & - & 1,09 \\
\hline Insetos adultos năo identificados & 1 & - & - & 1 & 1 & - & - & - & - & - & - & - & 3,29 \\
\hline Material não identificado & - & - & - & 1 & - & - & 2 & 1 & - & - & 1 & - & 5,58 \\
\hline Total & 6 & 4 & 7 & 8 & 6 & 6 & 8 & 11 & 7 & 9 & 13 & 6 & 100,0 \\
\hline
\end{tabular}

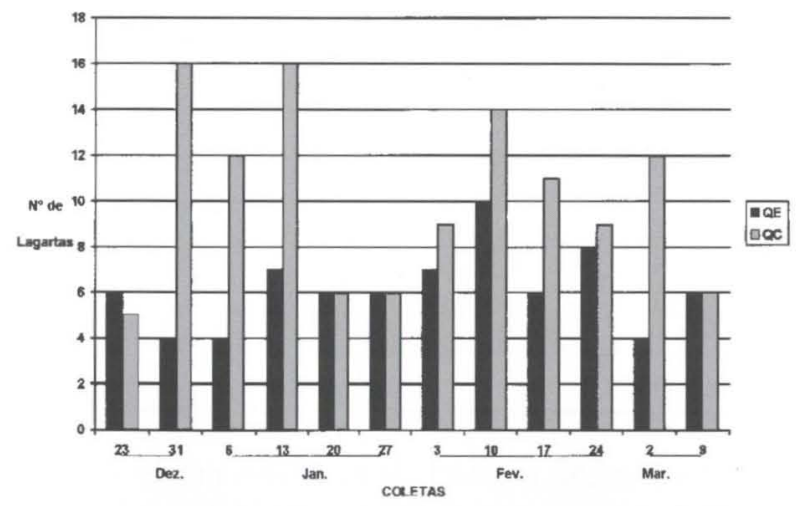

Fig. 1. Número de lagartas de Spodoptera frugiperda e Spdoptera latifascia encontradas em milho, nas quadras experimento $(\mathrm{QE})$ e controles $(\mathrm{QC})$, durante as coletas.

Algumas espécies como Heliothis virescens (15,38\%), Anticarsia gemmatalis (14,28\%), Trichoplusia ni (Huebner, 1802) (8,79\%) e Mocis latipes (Guenée, $1852)(1,09 \%)$, não foram amostradas na cultura do milho. Isso evidencia também a ação forrageadora das vespas fora da lavoura de milho, uma vez que essas espécies são encontradas em culturas adjacentes como, por exemplo, cana-de-açúcar, comprovando que as mesmas dirigem sua atividade de acordo com a oferta de recursos alimentares.

KIRKTON (1970) realizou um experimento de manipulação de colônias de vespas do gênero Polistes na predação de Heliothis zea (= Helicoperva zea) em uma lavoura de milho, mas as vespas concentraram sua atividade forrageadora sobre uma grande população de $S$. frugiperda atacando soja, comprovando a dificuldade de dirigir sua predação sobre uma determinada praga. 
O peso médio das presas trazidas por $P$. simillimus foi de $0,0158 \mathrm{~g}(0,0101$ $0,0553 \mathrm{~g}$ ), valor muito próximo aos obtidos também por Prezoto et al. (1994), que foi de $0,0134 \mathrm{~g}(0,0130-0,0399 \mathrm{~g})$.

$\mathrm{Na}$ figura 2, pode-se observar a quantidade de presas capturadas nas cinco colônias por cinco horas, nas diversas coletas semanais. A média de presas/hora trazidas pelas vespas foi calculada em 1,54, enquanto que o valor encontrado por PREZOTO et al. (1994) para uma colônia com uma população de 20 a 50 indivíduos, da mesma espécie foi de 1,7 presas/hora. KIRKTON (1970), verificou que as colônias de Polistes tinham de 10 a 13 vespas retornando com porções de lagartas por hora. Comparando-se com os valores obtidos no presente estudo, o mesmo corresponde à atividade forrageadora diária (12,32 presas/8 horas).

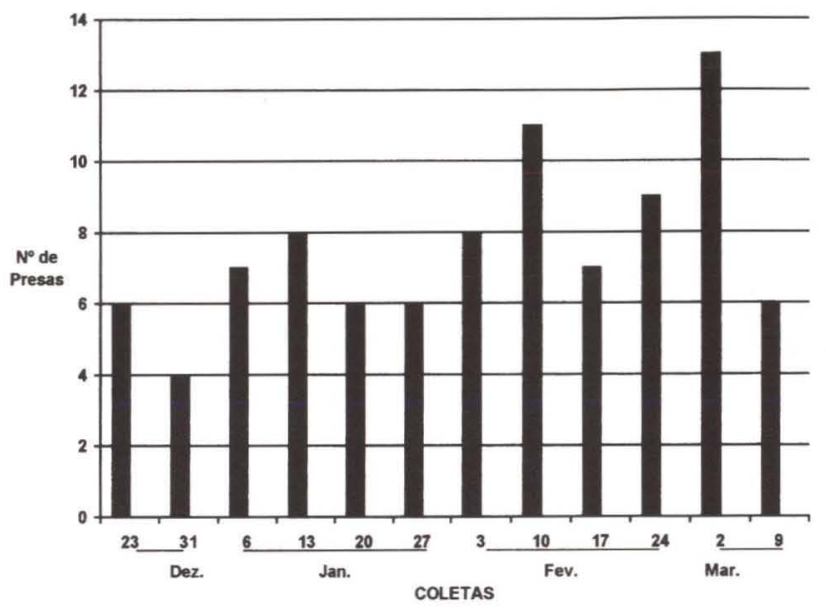

Fig. 2. Quantidade de presas capturadas por cinco colônias de Polistes simillimus durante cinco horas semanais na área experimental de uma cultura de milho durante as coletas.

Durante as coletas semanais de cinco horas em cinco colônias também se quantificou o número de retornos com presas, polpa de madeira, néctar e água, sendo os mesmos identificados pelo conteúdo visível (presa e polpa de madeira) ou por padrões comportamentais (néctar e água).

No decorrer das 12 coletas semanais (60 horas de observação) a quantificação de retornos foi de $40,61 \%$ com néctar, $30,17 \%$ com água, $21,57 \%$ com presas e 7,65\% com polpa de madeira. PrEzoto et al. (1994) estudando a atividade de um ninho de $P$. simillimus em estágio de pós-emergência, com cerca de 20 indivíduos, por um período de 11 horas (6:00 às 17:00 h), encontraram os seguintes valores: $36,46 \%$ retornos com água, $28,51 \%$ com néctar, $27,07 \%$ sem carga, 5,05\% com presas e $2,88 \%$ com polpa de madeira. Estes autores afirmam que esta maior porcentagem de retornos com água se deve ao fato das vespas controlarem a temperatura do ninho nas horas mais quentes do dia (das 13:30 às 15:00 horas), promovendo sua refrigeração. 
Aplicando-se o teste de correlação de Spearman encontrou-se uma correlação positiva entre o valor médio de presas/hora trazidas por $P$. simillimus com o tamanho da colônia ( $\mathrm{rs}=0,90 ; \mathrm{t}=3,57 \mathrm{e} \mathrm{p}=0,037$ ) (Tab. IV), o mesmo acontecendo para a correlação entre o número de presas e o tamanho do ninho $(r s=0,90 ; t=3,57$ e $\mathrm{p}=0,037)$.' Assim as colônias que foram bem sucedidas tais como: B, E e M apresentaram os maiores valores médios de presas/hora: 3,0,2,33 e 1,58, respectivamente. Aquelas que não tiveram um desenvolvimento satisfatório, tais como as colônias Q e U apresentaram índices menores, 0,58 e 0,25, respectivamente.

Tabela IV. Crescimento das colônias de Polistes simillimus translocadas para uma cultura de milho em 1996.

\begin{tabular}{|c|c|c|c|c|c|c|c|}
\hline & $\begin{array}{l}N^{\circ} \text { de células } \\
\text { em 22/XII/1995 }\end{array}$ & $\begin{array}{l}\mathrm{N}^{\circ} \text { de vespas em } \\
22 / \mathrm{X} \| / 1995\end{array}$ & Abandonos & $\begin{array}{l}N^{\circ} \text { de células } \\
\text { em 08/III/1996 }\end{array}$ & $\begin{array}{l}N^{\circ} \text { de vespas } \\
\text { em 08/III/1996 }\end{array}$ & $\begin{array}{l}\text { Indice de } \\
\text { crescimento } \\
\text { (célula/dia) }\end{array}$ & $\begin{array}{c}\text { Média de } \\
\text { captura de } \\
\text { presas/hora }\end{array}$ \\
\hline A & 19 & 4 & $19 / / 1996^{*}$ & 19 & 0 & 0,00 & - \\
\hline B & 165 & 13 & & 795 & 152 & 8,07 & 3,00 \\
\hline C & 51 & 3 & & 98 & 10 & 0,60 & - \\
\hline D & 64 & 7 & & 75 & 5 & 0,14 & - \\
\hline $\mathrm{E}$ & 103 & 8 & & 540 & 72 & 5,60 & 2,33 \\
\hline $\mathrm{F}$ & 82 & 9 & & 93 & 5 & 0,14 & - \\
\hline G & 184 & 12 & 05/I/1996 ** & 184 & 0 & 0,00 & - \\
\hline $\mathrm{H}$ & 73 & 8 & & 254 & 24 & 2,32 & - \\
\hline 1 & 134 & 6 & & 218 & 9 & 1,07 & - \\
\hline$J$ & 124 & 8 & $30 / X I 1 / 1995$ * & 124 & 0 & 0,00 & - \\
\hline $\mathrm{L}$ & 29 & 2 & $30 / X 11 / 1995^{*}$ & 29 & 0 & 0,00 & - \\
\hline M & 120 & 7 & & 281 & 63 & 2,06 & 1,58 \\
\hline $\mathrm{N}$ & 63 & 3 & & 102 & 7 & 0,50 & - \\
\hline 0 & 117 & 17 & $12 / 1 / 1996$ ** & 136 & 0 & 0,24 & - \\
\hline $\mathrm{P}$ & 3 & 1 & & 81 & 5 & 1,00 & - \\
\hline$Q$ & 150 & 8 & & 257 & 10 & 1,37 & 0,58 \\
\hline $\mathrm{R}$ & 3 & 2 & & 60 & 7 & 0,73 & - \\
\hline$S$ & 44 & 1 & & 139 & 15 & 1,21 & - \\
\hline$T$ & 79 & 3 & & 95 & 8 & 0,20 & - \\
\hline$U$ & 136 & 6 & & 271 & 20 & 1,73 & 0,25 \\
\hline
\end{tabular}

$\left(^{\star}\right)$ Ataque de formigas, $\left(^{\star \star}\right)$ Tempestade.

Das vinte colônias de $P$. simillimus translocadas obteve-se um sucesso de $75 \%$ na aceitação dos abrigos artificiais de madeira e, observou-se o abandono de 5 colônias ( $25 \%$ ), no decorrer do experimentos devido à tempestades (colônias G e $\mathrm{O}$ ) e ataque de formigas (colônias A, J e L). Esse sucesso obtido na translocação das colônias de P. simillimus é superior aos 60\% encontrado por BUTIGNOL (1992), num experimento envolvendo 30 colônias de $P$. versicolor.

Com relação ao aumento do tamanho do ninho, observa-se que as colônias B e E apresentaram os maiores índices de crescimento, 8,07 e 5,60 células/dia, respectivamente. Com exceção das colônias $\mathrm{H}$ e $\mathrm{M}$ que apresentaram índices consideráveis de crescimento $(2,32$ e 2,06), as demais colônias não superaram 1,73 células ao dia.

GiANNOTTI (1994) encontrou o valor de 7,4 células/dia, para o crescimento de uma colônia de $P$. simillimus no mesmo estágio.

GoBBI et al. (1993) realizaram um estudo comparativo da produtividade colonial entre $P$. simillimus e $P$. versicolor (Olivier, 1791) e, encontraram que a 
primeira espécie apresenta número de células $(391,3 \pm 302,34)$ e número de adultos produzidos por colônia $(144,0 \pm 150,2)$ muito superior ao da segunda espécie $(80,0$ $\pm 114,88$ e $22,0 \pm 36,9$, respectivamente). Além dessas diferenças, os autores também observaram que $P$. simillimus reutiliza somente uma pequena porcentagem das células de cria, enquanto que $P$. versicolor utiliza as células por até 3 gerações.

Comparando-se os dados obtidos entre quadras experimento e controles durante o desenvolvimento do milho, estimou-se para a área experimental $\left(700 \mathrm{~m}^{2}\right)$ uma redução na incidência da lagarta S. frugiperda em cerca de $77,16 \%$, e em $80 \%$ na população de Helicoperva zea (presente na espiga), correspondente na sua maioria à ação predatória de $P$. simillimus. I.A.F.S. (1976) constataram um resultado semelhante (70 a 80\%), no controle de Heliothis armigera (Huebner, 1802) em algodão, cinco a sete dias após a introdução de colônias de vespas Polistes em abrigos artificiais na área experimental.

As colônias de vespas, uma vez introduzidas em uma determinada área, crescem, multiplicam-se e disseminam-se pela região, favorecendo sua manutenção e continuada eficiência no controle biológico das pragas.

AGRADECIMENTOS. Ao Dr. Edilberto Giannotti pelas sugestões construtivas no decorrer do trabalho.

\section{REFERÊNCIAS BIBLIOGRÁFICAS}

BoiçA JR., A.L.; J.C. GALli; S.A. BorToli \& C. Rodrigues JR. 1992. Análise de danos produzidos por Spodoptera frugiperda (J.E. Smith, 1797) (Lepidoptera: Noctuidae) em cultivo de milho. Rev. Agric. 67 (2): 145-166.

Butignol, C.A. 1992. Observações sobre a bionomia da vespa predadora Polistes versicolor (Olivier, 1791) (Hymenoptera, Vespidae) em Florianópolis/SC. An. Soc. Entomol. Brasil. 19 (2): 201-206.

GianNotTi, E. 1994. Notes on the biology of Polistes simillimus Zikán (Hymenoptera, Vespidae). Bioikos. 8 (1/2):41-49.

GILLASPY, J.E. 1970. Experiments with papernest wasps for control of injurious caterpillars. S. Texas Agric. 1 (7): 20-21.

. 1971a. Papernest wasps (Polistes): observations and study methods. Ann. Entomol. Soc. Amer. 64 (6): 1357-1361.

. 1971b. Experiments with papernest wasps for caterpillar control in 1971. S. Texas Agric. 1 (8): 38-40.

. Polistes wasp studies in 1972. S. Texas Agric. 1 (9): 37-39.

1973a. Behavioral observations on paper-nest wasps (genus Polistes; family Vespidae; order Hymenoptera). Amer. Midl. Nat. 90 (1): 1-12.

—. 1973b. Wasp studies in 1973. S. Texas Agric. 1 (10): 89-91.

.1979. Management of Polistes wasps for caterpillar predation. Southwest. Entomol. 4 (4): 334-352.

Gobbi, N.; H.G. Fowler; J.C. NetTo \& S.L. Nazareth. 1993. Comparative colony productivity of Polistes simillimus and Polistes versicolor (Hymenoptera: Vespidae) and the evolution of paragyny in the Polistinae. Zool. Jb. Physiol. 97: 239-243. 
I.A.F.S. (Institute of Agricultural and Forestrial Sciences). 1976. A preliminary study on the bionomics of hunting wasps and their utilization in cotton insect control. Acta Entomol. Sinica. 19 (3): 303-308.

KIRKTON, R.M. 1970. Habitat management and its effect on population of Polistes and Iridomyrmex. Proc. Tall. Timbers Conf. 2: 243-246.

Morimoto, R. 1960a. Polistes wasps as natural enemies of agricultural and forest pests. I. (Studies on the social Hymenoptera of Japan. X). Sci. Bull. Fac. Agric. Kyushu Univ. 18: 109-116.

1960b. Polistes wasps as natural enemies of agricultural and forest pests. II. (Studies on the social Hymenoptera of Japan. XI). Sci. Bull. Fac. Agric. Kyushu Univ. 18: 117-132.

. 1961. Polistes wasps as natural enemies of agricultural and forest pests. III. (Studies on the social Hymenoptera of Japan. XII). Sci. Bull. Fac. Agric. Kyushu Univ. 18: 243-252.

NaKano, O.; S.S. NeTo \& R.A. ZuCCHI. 1981. Entomologia econômica. São Paulo, Livroceres, 314p.

Prezoto, F.; E. GianNotTI \& V.L.L. Machado. 1994. Atividade forrageadora e material coletado pela vespa social Polistes simillimus Zikán, 1951 (Hymenoptera, Vespidae). Insecta 3 (1): 11-19.

RABB, R.L. 1960. Biological studies of Polistes in North Carolina (Hymenoptera, Vespidae). Ann. Entomol. Soc. Amer. 53: 111-121.

RABB, R.L. \& F.R. LAWSON. 1957. Some factors influencing the predation of Polistes wasps on tobacco hornworm. Jour. Econ. Ent. 50: 778-784.

RICHARDS, O.W. \& M.J. RICHARDS. 1951. Observations on the social wasps of South America (Hymenoptera, Vespidae). Trans. Ent. Soc. Lond. 102: 1-170. TURILlazZI, S. 1980. Use of artificial nests for rearing and studying Polistes wasps. Psyche. 87 (1/2): 131-140.

ZUCCHI, R.A.; S.S. NETO \& O. NAKANO. 1993. Guia de identificação de pragas agrícolas. Piracicaba, Fealq, 139p.

Recebido em 02.IV.1998; aceito em 27.VIII.1999. 